(1)

CrossMark

\title{
New insights into immunological responses to infection in bronchiectasis
}

\author{
Michal Shteinberg ${ }^{1,3}$, Anand Shah ${ }^{2,3}$ and J. Stuart Elborn ${ }^{2}$
}

Affiliations: ${ }^{1}$ Pulmonology Institute and CF Center, Carmel Medical Center and the Technion-Israel Institute of Technology, the B. Rappaport Faculty of Medicine, Haifa, Israel. ${ }^{2}$ Royal Brompton and Harefield NHS Foundation Trust and Imperial College, London, UK. ${ }^{3}$ Both authors contributed equally to the manuscript.

Correspondence: Michal Shteinberg, Carmel Medical Center, Pulmonology Institute and CF Centre, 7 Michal St, Haifa 3463209, Israel. E-mail: michalshatechnion.ac.il

@ERSpublications

Immunological responses to infection are impaired in bronchiectasis. Autoimmune antibodies to neutrophil components may impair clearance of Pseudomonas and thus contribute to the chronicity of infection. http://ow.ly/MGm230n4AEF

Cite this article as: Shteinberg M, Shah A, Elborn JS. New insights into immunological responses to infection in bronchiectasis. Eur Respir J 2019; 53: 1802395 [https://doi.org/10.1183/13993003.02395-2018].

Infection and inflammation drive the pathogenesis of lung injury in bronchiectasis. The "vicious cycle" hypothesis suggests that infection leads to inflammation, which in turn disrupts normal bronchial structure and mucociliary clearance. This stimulates excessive mucus production which fosters bronchial infection causing further airway and parenchymal injury and further amplifying the intensity of inflammation.

Chronic infection with Pseudomonas aeruginosa (PA) occurs in 20-30\% of people with bronchiectasis [1] and $50 \%$ of adults with cystic fibrosis (CF). A key feature in both CF and bronchiectasis is that, unlike airway infections in individuals without bronchial pathology, infection with $P$. aeruginosa frequently does not resolve, but persists in a state of chronic infection. Chronic P. aeruginosa infection is associated with increased severity of disease, more frequent exacerbations and increased mortality in both diseases [2]. Some of the mechanisms that allow $P$. aeruginosa to evade the host immune response and persist in the airways involve alteration of the host immune function. Functional antibodies to $P$. aeruginosa develop in $\mathrm{CF}$ and bronchiectasis in response to $P$. aeruginosa infection, but rather than clearing the pathogen, they are associated with future $P$. aeruginosa infection in previously uninfected people with $\mathrm{CF}$, and with persistence of infection in both CF [3] and bronchiectasis $[4,5]$. While a defined immune deficiency is an established aetiology of bronchiectasis, it is only identified in a minority of patients (7-8\%) using standard agreed immune function tests [6].

Innate immunity has an important role in protection from bacterial pathogens. Bacterial permeability increasing protein (BPI), expressed in neutrophils and secreted in response to bacteria [7], binds to Gram-negative bacteria and disrupts their outer membrane, neutralises endotoxin, and through the C-terminal region opsonises bacteria [8]. BPI is expressed in intestinal epithelial cells, and its expression is enhanced in the gut following disruption of the intestinal mucosa [9]. BPI expression in the gut promotes the intestinal innate immune system, which is constantly exposed to multiple bacteria, to generate an antibacterial inflammatory response only when intestinal damage occurs. Interestingly, a genetic polymorphism of BPI is associated with an increased risk of inflammatory bowel disease (IBD) [10], suggesting that BPI modulates intestinal inflammation. 
In $\mathrm{CF}$ and bronchiectasis, a neutrophilic-dominated inflammation occurs in the airways, with markers of inflammation associated with increased severity [11]. For example, people with bronchiectasis and rheumatoid arthritis (RA) have elevated autoantibodies compared to people with RA without bronchiectasis [12], and increased severity of both RA and IBD is associated with worse lung function in individuals with associated bronchiectasis [13].

In the CF airways, anti-neutrophil cytoplasmic antibodies against BPI have been demonstrated to develop in individuals colonised with $P$. aeruginosa $[14,15]$. These antibodies are directed against the C-terminus of BPI, and inhibit the opsonisation and phagocytosis of E. coli [16]. Presence of anti-BPI antibodies are associated with persistence of $P$. aeruginosa and worse lung function in children with cystic fibrosis [14].

In this issue of the European Respiratory Journal, SKopelja-Gardner et al. [17] report their findings evaluating the relationship between anti-BPI autoimmunity and chronic $P$. aeruginosa infection. The authors show a strong association between anti-BPI antibodies and the presence of anti- $P$. aeruginosa antibodies (as a marker of current or previous $P$. aeruginosa infection) in two heterogeneous bronchiectasis cohorts. This relationship is further examined through a retrospective longitudinal study in a single bronchiectasis cohort showing a similar temporal relationship between BPI autoimmunity and a $P$. aeruginosa humoral response. Although a causal relationship is not proven, given the close, synchronised relationship, the authors hypothesise that the breaking of tolerance to BPI is mediated through an association with chronic $P$. aeruginosa infection.

A further study by the same group shows that in contrast to the anti-citrullinated protein autoantibodies generated in bronchiectasis patients with RA, the development of anti-BPI autoantibodies may relate to novel cryptic epitope formation [18]. Their research elegantly shows that BPI co-localises to neutrophil extracellular traps in CF patients with anti-neutrophil BPI auto-reactivity mapping to the C-terminal region of BPI without requiring post-translational modification (e.g. citrullination). They have further shown that BPI cleavage is $P$. aeruginosa elastase dependent, thus presenting a model of P. aeruginosa dependent BPI cleavage resulting in neo-epitope formation, activation of the adaptive immune system and triggering of autoimmunity.

The role of neutrophil extracellular traps in autoimmunity is of increasing interest, with inefficient clearance of micro-organisms contributing to the development of autoantibodies and driving the pathogenesis of systemic lupus erythematosus (SLE) [19]. In CF neutrophils have a pro-survival phenotype with reduced apoptosis and increased neutrophil extracellular trap formation [20]. Neutrophil elastase has likewise been shown to correlate with exacerbations and lung function decline in bronchiectasis [11]. Thus, the inefficient neutrophil predominant immune response seen in bronchiectasis may impair immune tolerance and generate an autoimmune response. This in turn may impair clearance of bacteria, perpetuating the infection-inflammation cycle (figure 1). Further studies, including B-cell cloning, epitope mapping and in vivo modelling, are required to confirm a mechanistic understanding of antibody

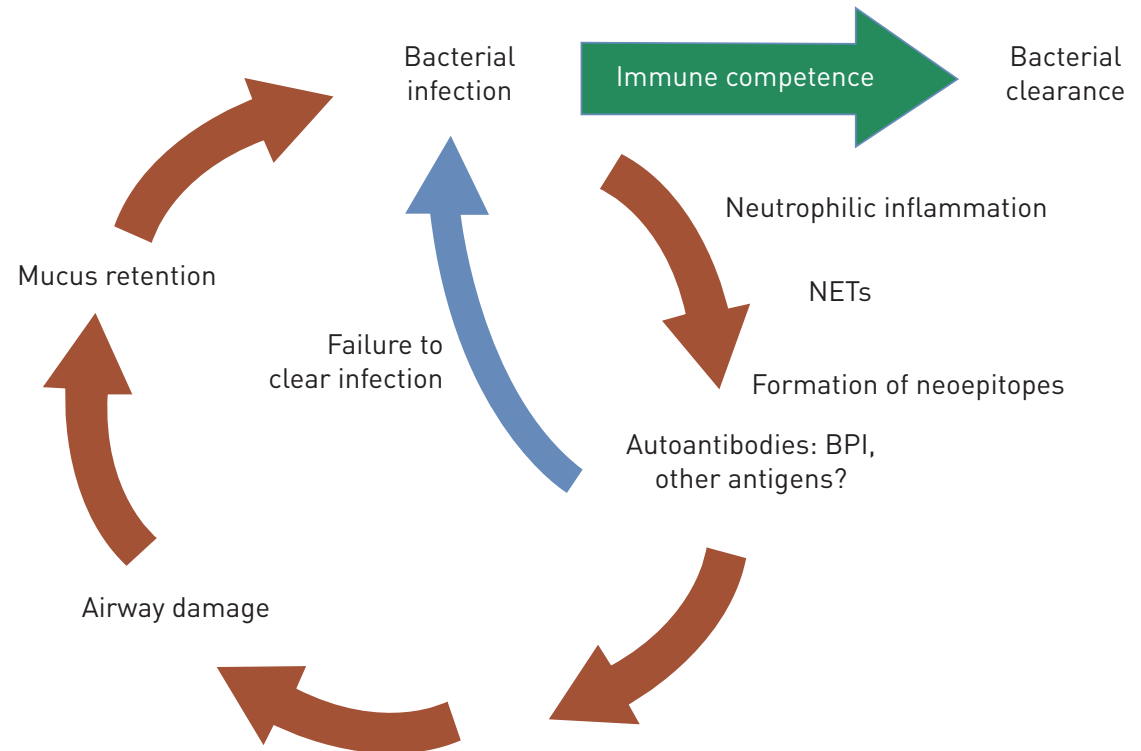

FIGURE 1 Inflammatory pathways promoting persistence of infection. NETs: neutrophil extracellular traps; $\mathrm{BPI}$ : bacterial permeability-increasing protein. 
formation in anti-BPI autoimmunity, but the emerging importance of autoimmunity in chronic lung infection presents potential future novel therapeutic strategies.

There are however some unanswered questions. First, it is unclear to what extent autoimmunity to BPI is driving pathogenesis in bronchiectasis. Secondly, are there further antigens resulting in autoimmunity in bronchiectasis and chronic lung infection that are driving disease progression and susceptibility to infection? Autoimmunity to interferon signaling pathways and cytokines have been well described but their relevance in chronic lung infection is undetermined [21,22]. Finally, could autoimmunity reversal with B-cell targeted therapy either through pharmacological, immunotherapy or cell-based therapy halt disease progression in bronchiectasis, or would such treatments be deleterious if the function of neutrophils that partially control infection is disturbed? The development of in vivo models would enable testing of these questions. A simpler and targeted approach, however, already in trial would be to target neutrophil elastase directly to reduce neo-epitope formation and autoimmunity triggering. Analysis of autoimmunity therefore may be an important secondary outcome measure in neutrophil-targeted clinical trials.

The primary insult in bronchiectasis, which initiates the "vicious cycle" is, in most cases, unknown. Current research findings suggest that autoimmunity to neutrophil components disturb neutrophil function, allowing persistence of infection. These autoimmune phenomena may develop in response to infection, and result in an inability to overcome infection. There is hence increasing importance of immune phenomena in the aetiology and persistence of infection in bronchiectasis, whilst the complex mechanisms remain to be elucidated.

Conflict of interest: M. Shteinberg reports having received research grants from GSK, Novartis, Trudell Pharma and GSK; travel grants from Novartis, Actelion, Boeringer Ingelheim, GSK and Rafa; speaker fees from Boehringer Ingelheim, GSK, AstraZeneca, Teva and Novartis; advisory fees from GSK, Boehringer Ingelheim, Kamada and Horizon Pharma; travel grants from GSK, Boehringer Ingelheim and Rafa. A. Shah has nothing to disclose. J.S. Elborn reports grants from FP7, Innovative Medicines Inititive, and the UK Government (NIHR), outside the submitted work.

\section{References}

1 Finch S, McDonnell MJ, Abo-Leyah H, et al. A Comprehensive analysis of the impact of Pseudomonas aeruginosa colonization on prognosis in adult bronchiectasis. Ann Am Thorac Soc 2015; 12: 1602-1611.

2 Araújo D, Shteinberg M, Aliberti S, et al. The independent contribution of Pseudomonas aeruginosa infection to long-term clinical outcomes in bronchiectasis. Eur Respir J 2018; 51: 1701953.

3 Mauch RM, Nørregaard LL, Ciofu O, et al. IgG avidity to Pseudomonas aeruginosa over the course of chronic lung biofilm infection in cystic fibrosis. J Cyst Fibros 2018; 17: 356-359.

4 Suarez-Cuartin G, Smith A, Abo-Leyah H, et al. Anti-Pseudomonas aeruginosa IgG antibodies and chronic airway infection in bronchiectasis. Respir Med 2017; 128: 1-6.

5 Caballero E, Drobnic ME, Pérez MT, et al. Anti-Pseudomonas aeruginosa antibody detection in patients with bronchiectasis without cystic fibrosis. Thorax 2001; 56: 669-674.

6 Araújo D, Shteinberg M, Aliberti S, et al. Standardised classification of the aetiology of bronchiectasis using an objective algorithm. Eur Respir J 2017; 50: 1701289.

7 Elsbach P. The bactericidal/permeability-increasing protein (BPI) in antibacterial host defense. J Leukoc Biol 1998; 64: 14-18.

8 Levy O, Elsbach P. Bactericidal/permeability-increasing protein in host defense and its efficacy in the treatment of bacterial sepsis. Curr Infect Dis Rep 2007; 3: 407-412.

9 Balakrishnan A, Chakravortty D. Epithelial cell damage activates bactericidal/permeability increasing-protein (BPI) expression in intestinal epithelium. Front Microbiol 2017; 8: 1567.

10 Fan $\mathrm{L}, \mathrm{Fu} \mathrm{G}$, Ding Y, et al. Bactericidal/permeability increasing protein gene polymorphism and inflammatory bowel diseases: meta-analysis of five case-control studies. Int J Colorectal Dis 2017; 32: 433-435.

11 Chalmers JD, Moffitt KL, Suarez-Cuartin G, et al. Neutrophil elastase activity is associated with exacerbations and lung function decline in bronchiectasis. Am J Respir Crit Care Med 2017; 195: 1384-1393.

12 Perry E, Eggleton P, De Soyza A, et al. Increased disease activity, severity and autoantibody positivity in rheumatoid arthritis patients with co-existent bronchiectasis. Int J Rheum Dis 2015; 20: 2003-2011.

13 Goeminne PC, Verschueren P, Scheers H, et al. Safety of immunomodulatory therapy in patients with bronchiectasis associated with rheumatic disease and IBD: a retrospective and cohort analysis. Clin Rheumatol 2012; 31: 367-373.

14 Zhao MH, Jayne DR, Ardiles LG, et al. Autoantibodies against bactericidal/permeability-increasing protein in patients with cystic fibrosis. QJM 1996; 89: 259-265.

15 Sedivá A, Bartůnková J, Kolárová I, et al. Antineutrophil cytoplasmic autoantibodies (ANCA) in children with cystic fibrosis. J Autoimmun 1998; 11: 185-190.

16 Mahadeva R, Dunn AC, Westerbeek RC, et al. Anti-neutrophil cytoplasmic antibodies (ANCA) against bactericidal/permeability-increasing protein (BPI) and cystic fibrosis lung disease. Clin Exp Immunol 1999; 117: 561-567.

17 Skopelja-Gardner S, Theprungsirikul J, Meagher RE, et al. Autoimmunity to bactericidal/permeability-increasing protein in bronchiectasis exhibits a requirement for Pseudomonas aeruginosa IgG response. Eur Respir J 2019; 53: 1801891.

18 Skopelja S, Hamilton BJ, Jones JD, et al. The role for neutrophil extracellular traps in cystic fibrosis autoimmunity. JCI Insight 2016; 1: e88912. 
19 Apel F, Zychlinsky A, Kenny EF. The role of neutrophil extracellular traps in rheumatic diseases. Nat Rev Rheumatol 2018; 14: 467-475.

20 Gray RD, Hardisty G, Regan KH, et al. Delayed neutrophil apoptosis enhances NET formation in cystic fibrosis. Thorax 2018; 73: 134-144.

21 Lin C-H, Chi C-Y, Shih H-P, et al. Identification of a major epitope by anti-interferon- $\gamma$ autoantibodies in patients with mycobacterial disease. Nat Med 2016; 22: 994-1001.

22 Vincent T, Plawecki M, Goulabchand R, et al. Emerging clinical phenotypes associated with anti-cytokine autoantibodies. Autoimmun Rev 2015; 14: 528-535. 